\title{
Clinical Diagnosis and Treatment Characteristics of Acute Respiratory Infections in Children and New Developments in Laboratory Testing
}

\author{
Yueliao Ma1, Lingyu Lü ${ }^{2 *}$ Qiangcai $\mathrm{Mai}^{{ }^{*}}$ \\ ${ }^{1}$ Laboratory Department of Maternity and Child Health Hospital of Yao Autonomous County, Hechi, China \\ ${ }^{2}$ Laboratory Department of Traditional Chinese Medicine Hospital of Nandan County, Hechi, China \\ ${ }^{3}$ Second Department of Internal Medicine, Orthopedic Hospital of Integrated Traditional Chinese and Western Medicine, \\ Guigang, China \\ Email:56835477@qq.com, ${ }^{*} 18007856271 @ 163 . c o m,{ }^{*} 530653796 @ q q . c o m$
}

How to cite this paper: Ma, Y.L., Lu, L.Y. and Mai, Q.C. (2021) Clinical Diagnosis and Treatment Characteristics of Acute Respiratory Infections in Children and New Developments in Laboratory Testing. Open Journal of Pediatrics, 11, 114-124. https://doi.org/10.4236/ojped.2021.111011

Received: February 9, 2021

Accepted: March 12, 2021

Published: March 15, 2021

Copyright ( 2021 by author(s) and Scientific Research Publishing Inc. This work is licensed under the Creative Commons Attribution International License (CC BY 4.0).

http://creativecommons.org/licenses/by/4.0/

\begin{abstract}
Acute respiratory tract infection (ARTI) in children is the most common infectious disease in childhood, and its pathogens include viruses, bacteria and fungi, mycoplasma, chlamydia and rickettsia. In recent years, with the continuous development of pathogen detection methods, the diagnosis and treatment of acute respiratory infections has received more and more clinical attention. The clinical diagnosis and treatment characteristics of acute respiratory infections in children and the research of clinical laboratory detection methods have also been continuously developed. The author collected references to review the clinical features and new developments in laboratory testing of acute respiratory tract infection in children.
\end{abstract}

\section{Keywords}

Children, Respiratory Tract Infections, Pathogens, Laboratory Testing, Acute

\section{Introduction}

Acute respiratory tract infection in children is commonly known as pediatric cold, also known as "cold". It is a common cold, also known as acute nasopharyngitis which is the most common disease in acute upper respiratory tract viral infections. It is mostly self-limiting, but the incidence is high, affecting the wide 
population and economic losses are considerable, and can cause many complications [1] [2]. The complications of acute nasopharyngitis include purulent pharyngitis, sinusitis, otitis media, bronchitis, acute exacerbation of the original chronic respiratory diseases (chronic bronchitis, asthma), and worsening of obstructive sleep disordered breathing. Children occasionally have serious complications such as viral or bacterial pneumonia. Acute respiratory infections in children refer to acute infections above the throat, also known as upper respiratory tract infections, which are the most common infectious diseases in children. The disease mainly affects the nose, nasopharynx and pharynx. $90 \%$ of children's colds are caused by viruses, and can also be caused by bacteria or mycoplasma infections. Children's colds can occur throughout the year, more often in winter and spring. Infants and young children are prone to this disease due to the anatomy and immune characteristics of the upper respiratory tract. Therefore, there are more cases in early childhood, and gradually decrease in school-age children. Due to the difference of age, physical strength and lesion location, the priority and severity of the disease are also different [3] [4] [5]. In order to understand the clinical diagnosis and treatment characteristics of children with acute respiratory tract infections and the new progress of laboratory testing, the author collected references to review the clinical features and new developments in laboratory testing of acute respiratory tract infection in children.

\section{Symptoms of Acute Respiratory Infections in Children}

The incubation period of acute respiratory infections in children ranges from 1 to 3 days, and varies with the virus; enteroviruses are the shortest, and adenovirus and respiratory syncytial virus are longer; The incubation period of acute respiratory infections in children ranges from 1 to 3 days, and varies with the virus. Enteroviruses are the shortest, and adenovirus and respiratory syncytial virus are longer. The onset is sudden. Most people experience a burning sensation in the nose and throat, followed by nasal congestion, sneezing, runny nose, general malaise, and muscle aches. Symptoms reach a peak at 48 hours (virus uncoating). Acute nasopharyngitis usually has no fever or only low fever, especially when rhinovirus or coronavirus infection occurs. There may be conjunctival hyperemia, tearing, photophobia, eyelid swelling, and throat mucosal edema. The presence of throat and tracheitis varies from person to person and from virus to virus. Nasal secretions are initially a lot of watery clear nose, and later become mucus or purulent. Mucopurulent discharge does not necessarily indicate a secondary bacterial infection. Coughing is usually not severe and can last up to 2 weeks. Purulent sputum or severe lower respiratory tract symptoms suggest that viruses other than rhinoviruses are combined or secondary to bacterial infections. When children catch a cold, their symptoms are more severe than adults, and they often have lower respiratory symptoms and gastrointestinal symptoms (vomiting, diarrhea, etc.). Colds are mostly self-limiting, if there are no complications, the course of the disease is 4 to 10 days [6] [7] [8]. 


\section{Pathogenesis of Acute Respiratory Infections in Children}

The rhinovirus, the pathogen of acute respiratory infections in children, is mainly caused by contact transmission (hand-eye, hand-nose) caused by the contamination of the nasopharyngeal secretions of cold patients, and there are also droplet transmission routes; the latter is far less important in acute nasopharyngitis than influenza [9] [10] [11] [12]. After rhinovirus infection, virus replication reaches a peak concentration at 48 hours, and the transmission period lasts for 3 weeks. Individual susceptibility is related to nutritional health status, upper respiratory tract abnormalities (such as tonsil enlargement) and smoking; the cold itself does not cause a cold. Part of the reasons for common cold in cold season are related to the type of virus, and may also be related to the increase of indoor family members or crowd aggregation and crowding caused by cold. The symptoms of infection are affected by the physiological status of the host. Overwork, depression, allergic diseases of nasopharynx and menstruation can aggravate the symptoms. Take rhinovirus as an example, the nasal cavity or eyes are the gateway to the body, and the nasopharynx is the initial site of infection. M cells in the lymphoepithelial region of adenoids contain the receptor for intercellular adhesion molecule-1 (ICAM-1) of rhinovirus, where the virus first adheres and then reaches the posterior nasopharynx by mucociliary activity in the nasal cavity. At this time, the virus replicates rapidly and spreads forward to the nasal meatus. The study of nasal epithelial cell biopsy and nasal secretion indicated that the secretion of inflammatory mediators (bradykinin, prostaglandin), interleukin-1 and interleukin-8 increased, which may be partly responsible for the clinical symptoms of cold. The role of histamine is unclear. Although intranasal instillation of histamine can cause cold symptoms, the therapeutic effect of antihistamine drugs is uncertain. Parasympathetic blockers are effective in relieving the symptoms of common cold, suggesting that the mechanism of nerve reflex also plays a role in the pathogenesis of common cold. The immune response (IgA, interferon production) is usually short-lived, coupled with the diversity and drift of viral antigens, so multiple infections are repeated in a lifetime. The pathological changes are related to the virulence and infection range of the virus. Respiratory tract mucosa is edema, congestion, and exudation (leakage or exudation), but there is no important change in the cell population, repair is relatively rapid, and generally does not cause tissue damage. Different viruses can cause different degrees of cell proliferation and degeneration. The duration of mucociliary destruction is 2 - 10 weeks. When the infection is serious, the nasal sinuses, Eustachian tube and middle ear canal may be blocked, resulting in secondary infection.

\section{Pathological Etiology of Acute Respiratory Tract Infection in Children}

The pathogens of acute respiratory tract infection in children are viruses; the common ones are rhinovirus, coronavirus, influenza and parainfluenza viruses; the rare ones include respiratory syncytial virus, adenovirus, enterovirus, reovi- 
rus, herpes simplex virus and E-B virus etc. Mycoplasma pneumoniae, group A, $\mathrm{C}, \mathrm{G}$ streptococcus and abiotic pathogenic factors such as allergic rhinitis, atrophic rhinitis, vasomotor rhinitis, deviation of nasal septum, foreign bodies can cause cold like symptoms, which is not a real cold [13]-[18]. 1) Rhinovirus Rhinovirus was isolated from cold patients in 1956. It is a genus of microRNA virus group. It is a single stranded RNA virus without envelope, with a diameter of 15 - 30 $\mathrm{nm}$. It is resistant to ether, but not acid (it is easy to be inactivated in $\mathrm{pH} 3$ solution). It can survive for 3 days in dry environment. According to the serum neutralization test, there are more than 120 serum strains. Diploid cells grow best at $33^{\circ} \mathrm{C}$. They can survive for a long time at $-70^{\circ} \mathrm{C}$ and for several weeks at $4{ }^{\circ} \mathrm{C}$. They can be inactivated at $56^{\circ} \mathrm{C}$ for 30 minutes. $30 \%-50 \%$ of the common cold patients were caused by a certain serotype of rhinovirus. 2) Coronavirus Coronavirus belongs to coronavirus group. It is a single stranded RNA virus with envelope. It is sensitive to ether and acid. They are $80-150 \mathrm{~nm}$ in diameter and have unique rod-shaped envelope granules protruding from the capsule, proliferating in the cytoplasm and maturing through the budding of cytoplasmic reticulum. At least three strains (B814,229E and 0C43) can cause respiratory tract infection. Among them, 229E and OC43 are the causes of upper respiratory tract infection in children and adults, accounting for $15 \%-20 \%$ of the total number of influenza in adults. Occasionally, they can also cause pneumonia and influenza chest pain. 3) Adenovirus Adenovirus is a double stranded DNA virus with no envelope and replication in the nucleus. It has a diameter of $70-90 \mathrm{~nm}$. It is a symmetrical 20 hedron and is stable at $-20^{\circ} \mathrm{C}$. 41 serum strains have been isolated from human beings, and there are many intermediate strains. The clinical manifestations are various types of infection. 4) Respiratory syncytial virus Respiratory syncytial virus was first isolated in a laboratory from a chimpanzee suffering from an upper respiratory tract infection in 1956. It is an enveloped single-stranded RNA virus with a diameter of $120-200 \mathrm{~nm}$ and belongs to the Pneumovirus genus of the Paramyxoviridae family. There is only one serotype. The virus is extremely unstable. The amount of virus is reduced by 100 times in 2 days at room temperature, and it is also reduced by 100 times in 4 to 6 days in a refrigerator at $4^{\circ} \mathrm{C}$. It is the main pathogen of lower respiratory tract infections in children. It usually causes mild upper respiratory tract infections in adults, but it is more critically ill in the elderly and immunosuppressed patients. 5) Other viruses Coxsackievirus, echovirus, reovirus $1-3$, herpes simplex virus 1 and Epstein Barr virus in enterovirus may occasionally be used as pathogens of common cold.

\section{Disease Diagnosis of Acute Respiratory Infection in Children}

Acute respiratory infections in children need to be differentiated from other related diseases [19] [20] [21] [22], and to differentiate from influenza: 1) epidemiological influenza is a widespread epidemic disease, mainly in winter and 
spring, and acute nasopharyngitis occurs throughout the year, mostly in autumn, winter and spring. 2) Clinical manifestations: influenza systemic poisoning symptoms are obvious, often high fever, $39^{\circ} \mathrm{C}-40^{\circ} \mathrm{C}$, lasting for $3-5$ days, headache and systemic pain are common and serious, fatigue and weakness appear early, lasting for 2 - 3 weeks, can be accompanied by nasal congestion, sneezing, sore throat, chest discomfort and cough are common, and the degree is serious, can be complicated with bronchitis, pneumonia, and even life-threatening. Tracheitis and granular changes of soft palate mucosa are typical catarrhal symptoms of influenza. The main manifestations of acute nasopharyngitis are nasal congestion, sneezing, sore throat, mild systemic symptoms, headache, systemic pain and fatigue, less and mild weakness, less fever, some of them may have mild to moderate chest discomfort and cough, and complications are rare. 3) Auxiliary examination: a) X-ray examination: influenza is more prone to pulmonary complications than acute nasopharyngitis, such as bronchitis or pneumonia, so chest X-ray examination is helpful to the tendency of clinical diagnosis. b) Laboratory examination: i) Virus isolation and culture, by collecting nasal secretions, nasopharyngeal swabs, and throat swabs for furunculosis isolation and culture to determine the pathogen. The virus is cultured for about $3-10$ days. ii) Rapid influenza determination, sensitivity $>70 \%$, specificity $>90 \%$, about $24 \mathrm{~h}$. iii) For the detection of serum antibody, this method needs to determine the antibody titer in the acute and recovery phases. iv) In the examination of inferior turbinate mucosal imprint, a large number of degenerated columnar epithelial cells often appear in patients with influenza from the first day of onset, and there are aniline blue inclusions in the cytoplasm or nucleus. In acute nasopharyngitis, purulent cells are the main type, and inclusion bodies are rare.

Differentiation of acute respiratory infections and nasal diseases in children: 1) allergic rhinitis: allergic history, seasonal (pollinosis) or perennial sneezing, rhinorrhea, nasal congestion with itching. The characteristics of symptoms and the increase of eosinophils in nasal secretions are helpful for the diagnosis of the disease. 2) Vasomotor rhinitis: no allergic history, with nasal mucosa intermittent vascular filling, sneezing and runny nose as the characteristics, dry air can make the symptoms worse. It can be distinguished from viral infection or bacterial infection according to the history and absence of pus, mucus and crust. 3) Atrophic rhinitis: The nasal cavity is abnormally unobstructed, the lamina propria of the mucosa is thinned and the blood vessels are reduced, the sense of smell is reduced, and there is crust formation and foul smell, which is easy to distinguish. 4) Deviation of nasal septum and nasal polyps: the diagnosis can be confirmed by rhinoscopy.

In addition, acute respiratory infections in children need to be differentiated from certain acute infectious diseases (such as measles, encephalitis, epidemic cerebrospinal meningitis, polio, typhoid fever, and typhus) and upper respiratory tract inflammation in the prodromal period of HIV infection; according to 
the history of symptoms, dynamic observation and relevant laboratory examination, it is not difficult to identify.

\section{Laboratory Examination of Acute Respiratory Infections in Children}

The laboratory test for acute respiratory infections in children is firstly a peripheral blood test. The white blood cell count is normal or low during viral infection. The proportion of lymphocytes is increased. In bacterial infections, the proportion of white blood cells increases and the nucleus shifts to the left. The proportion of neutrophils increased and the nucleus shifted to the left. The second is pathogenic examination, which is not usually done. When necessary, immunofluorescence, enzyme-linked immunosorbent assay, serological diagnosis or virus isolation and identification methods can be used to determine the type of virus; bacterial culture and drug sensitivity tests are helpful for the diagnosis and treatment of bacterial infections. Finally, other auxiliary examinations can be done, such as chest X-ray examination, and there is no abnormality [23] [24] [25] [26].

\section{Treatment Plan for Acute Respiratory Infection in Children}

The treatment of acute respiratory infections in children is first commonly used with symptomatic treatment drugs [27]-[38]: 1) ipratropium bromide (ipratropium bromide) spray: placebo-controlled trials have proved effective in relieving runny nose and sneezing, especially on the first day of the early course. The main adverse reactions were nasal mucus with blood streak in $15 \%-20 \%$ patients. 2) Pseudoephedrine: It acts on $\alpha$-adrenergic receptors in the respiratory mucosa, relieves nasal mucosal congestion, and has little effect on the heart and other peripheral blood vessels $\alpha$-receptors. Reduce nasal congestion and improve sleep. It is not suitable for long-term application, but for 3 to 5 days. 3) Antihistamines: the first generation of antihistamines, such as chlorphenamine maleate (chlorphenamine), are effective in reducing sneezing and rhinorrhea. The elderly with prostatic hypertrophy should be cautious. Non sedative antihistamines lack anticholinergic effect and the effect is uncertain. 4) Antipyretic and analgesic: It is optional for patients with fever, muscle aches and headaches. Acetaminophen (paracetamol) is the most commonly used; it should be avoided to use it together with the anti HIV drug zivdodine. Repeated application of aspirin will increase the amount of virus excretion, but the effect of improving symptoms is slight, so it is not recommended. 5) Antitussive: In order to protect the cough reflex, it is generally not recommended to use it, but it can be used as appropriate when a severe cough affects the rest; dextromethorphan is more used. Followed by drugs or treatments that may be useful: 1) Vitamin C: The effect is uncertain. It has been reported that taking high-dose vitamin $\mathrm{C}(8.0 \mathrm{~g} / \mathrm{d})$ from the first day of infection can shorten the duration of symptoms and reduce the condition. But 
most authors hold a negative attitude towards this. 2) Zinc gluconates: In vitro experiments have shown its inhibitory effect on rhinovirus. Clinical controlled trials have shown that the duration of symptoms is shortened, but the results are very inconsistent and there are many adverse reactions. 3) Breathing and heating humidified gas: because the most suitable temperature for rhinovirus replication is $33^{\circ} \mathrm{C}$, it is advocated to treat cold by respiratory heating humidification gas. Another antiviral drug treatment: Ribavirin (ribavirin) has a certain inhibitory effect on influenza, parainfluenza virus, and respiratory syncytial virus. The clinical application is limited to children's lower respiratory tract infection with respiratory syncytial virus. There are no effective antiviral drugs for rhinovirus and other respiratory viruses. Finally, the application of antibiotics: Generally, antibiotics should not be and need not be applied. A placebo-controlled study showed that antibiotic treatment showed improvement in symptom scores in a subgroup (20\%) of patients with growth of Streptococcus pneumoniae, Haemophilus influenzae, or Moraxella catarrhalis in nasal and laryngeal lavage cultures. Therefore, in patients with bacterial colonization, increased granulocytes in respiratory secretions, sinusitis, otitis media and other complications, chronic obstructive pulmonary disease (COPD) and other basic diseases, and a cold course of more than one week, antibiotics can be used appropriately. In addition, preventive care is also more important to avoid contact with cold patients, especially hands. Experimental studies have reported that the use of virus-killing paper towels and maintaining good personal hygiene can reduce the spread of rhinovirus colds. Vitamin $\mathrm{C}$ is often advocated for the prevention of colds, but strictly designed controlled trials have not received supporting evidence. Interferon $\alpha-2 b$ has a preventive effect after exposure, but it caused adverse reactions such as nasal congestion, and the study was stopped.

\section{Summary and Outlook}

Acute respiratory infections in children are mainly caused by respiratory pathogens. Atypical respiratory pathogens refer to pathogens other than Streptococcus pneumoniae, including Legionella pneumophila (LP), Mycoplasma pneumoniae (MP), Chlamydia pneumoniae (CP), Q fever rickettsiae (QFR), adenovirus (ADV), Respiratory syncytial virus (RSV), influenza virus (IFV) and parainfluenza virus (PIV), etc. These pathogens mutate quickly and spread widely, and it is difficult to form long-term immunity in the body, so it is difficult to prevent and control. Bacteriological examination can be carried out by sputum culture or six-unit inspection of respiratory pathogens. In the laboratory diagnosis method of viral respiratory diseases, the direct immunofluorescence method for antigen detection takes a short time, and the results can be obtained within 2 hours of the specimens sent to the laboratory. It can be carried out in ordinary laboratories. Compared with other methods, it has the characteristics of quickness, simplicity, specificity and high sensitivity, and it is suitable for the selection of primary medical institutions [39] [40] [41]. 
Acute respiratory infections in children have high morbidity and mortality. Viral infection is the main pathogen of ARI. The clinical manifestations of ARI such as fever, sore throat, cough, fatigue, etc. lack specificity. There are few vaccines currently available to prevent respiratory virus infections. Therefore, understanding the pathogenic epidemiological characteristics of acute respiratory infections is of great significance to the effective prevention and control of virus epidemics in the future; choosing rapid and sensitive laboratory testing methods and early identification of ARI pathogens is for clinicians to determine disease diagnosis and select reasonable diagnosis and treatment plans which has guiding significance.

\section{Acknowledgements}

During the process of this topic research, we got much help from many departments and individuals, and other personnel not involved in this project research. All of them offered a great support and help in this research. Now here, all of members in this research show our deepest appreciation to them, and wish them good health and everything goes well.

\section{Funding}

Scientific Research and Technology Development Project of Hechi City, Guangxi (No.: Heke Gong 125504).

\section{Conflicts of Interest}

The authors declare no conflicts of interest regarding the publication of this paper.

\section{References}

[1] Wang, S.Y., Zhang, T.T., Zhang, X.M., et al. (2020) Epidemiological Characteristics and Economic Burden of Acute Respiratory Diseases in Children under 5 Years Old in Gansu Area. China Maternal and Child Health Care, 35, 2548-2552.

[2] Zhao, Y.Y. (2020) The Value of C-Reactive Protein and Blood Routine Testing in Children with Acute Upper Respiratory Tract Infection. Contemporary Medicine, 26, 140-142.

[3] Hu, S.Y. (2020) The Value of Combined Application of High-Sensitivity C-Reactive Protein and Blood Routine in the Diagnosis of Acute Upper Respiratory Tract Infection in Children. Great Doctor, 5, 46-48.

[4] Ma, H.L. and Ning, L.F. (2020) The Value of Serum C-Reactive Protein, B-Type Brain Natriuretic Peptide, Procalcitonin and Prealbumin in the Diagnosis and Efficacy Evaluation of Children with Acute Respiratory Infection. Journal of North Sichuan Medical College, 35, 52-56.

[5] Pan, H.Y., Lu, H.T. and Liu, C.J. (2020) The Diagnostic Value of Serum Procalcitonin and C-Reactive Protein in Children with Acute Upper Respiratory Tract Infection. Heilongjiang Medicine, 33, 690-692.

[6] Li, Z.Z. (2019) Analysis of Clinical Characteristics of Children with Acute Upper Respiratory Tract Infection in Nanning Area. Chinese Community Physician, 35, 
$150,153$.

[7] Wang, Z.X. (2019) The Distribution Characteristics and Treatment Suggestions of Pathogenic Bacteria in Children with Acute Upper Respiratory Tract Infection. Clinical Medical Research and Practice, 4, 73-74.

[8] Cheng, Y. (2019) The Use and Value Analysis of Antibacterial Drugs for Acute Upper Respiratory Tract Infections in Pediatric Outpatient Clinics. Lingnan Journal of Emergency Medicine, 24, 33-36.

[9] Ma, D.Q. and Wang, H. (2019) Etiological Investigation of Common Acute Respiratory Infections in Children in 2018. Chinese Community Physician, 35, 52, 54.

[10] Xiong, R.Z. (2019) Analysis of the Diagnosis and Treatment of Acute Respiratory Infections in Children with Bronchial Asthma. Chinese Continuing Medical Education, 11, 138-140.

[11] Wei, W.Y. (2019) Analysis of Respiratory Tract Etiology and Clinical Characteristics of 520 Cases of Acute Exacerbation of Bronchial Asthma in Children. China Modern Medicine Application, 13, 18-19.

[12] Ma, J.H. (2020) The Etiology and Clinical Treatment of Repeated Respiratory Infections in Children. China Health Care and Nutrition, 30, 89.

[13] Sun, B. (2020) Analysis of Detection of Common Respiratory Viruses in Acute Respiratory Infections in Children. Chinese Health Standard Management, 11, 108-111.

[14] Qi, C.R., Zhou, X.H., Zhang, Z.X., et al. (2019) Analysis of 3192 Cases of Epstein-Barr Virus Serology and Nucleic Acid Detection Results in Children with Respiratory Tract Infectious Diseases. Chinese Medicines and Clinics, 19, 1054-1056.

[15] Du, H.M., Rong, S.H., Lu, H.X., et al. (2019) The Clinical Value of Combined Application of Immunological Indicators, High-Sensitivity C-Reactive Protein (hs-CRP), and Blood Routine in the Diagnosis of Acute Upper Respiratory Tract Infection in Children. Clinical Research, 27, 41-43.

[16] Li, L. and Wang, Z.H. (2019) The Clinical Value of Combined Detection of C-Reactive Protein and Blood Routine in Children with Acute Upper Respiratory Tract Infection. Journal of Clinical Rational Use, 12, 144-145.

[17] Liu, Y. (2020) The Clinical Value of Procalcitonin and C-Reactive Protein Detection in Children with Acute Respiratory Infection. World Compound Medicine, 6, 33-35.

[18] Zhou, G.M. (2020) Study on the Application of White Blood Cell and CRP in the Diagnosis of Acute Upper Respiratory Tract Infection in Children. Diet and Health Care, 7, 239.

[19] Zhu, L.J. and Li, X.J. (2020) Application of Nucleic Acid Detection of Respiratory Pathogens in Diagnosis of Acute Respiratory Diseases in Children. Shenzhen Journal of Integrated Traditional Chinese and Western Medicine, 30, 53-55.

[20] Shen, X.Y. and Zou, S.C. (2020) Epidemiological Analysis of Mycoplasma Pneumonia Infection in Children with Respiratory Tract. Electronic Journal of Clinical Medicine, 7, 173.

[21] Han, L. and Wu, Y.L. (2020) Application Analysis of Serum PCT Combined with Blood Routine in the Differential Diagnosis of Acute Upper Respiratory Tract Infection in Children. Health Care Guide, No. 14, 273.

[22] Cai, X.X. (2020) Application Value of Combined Detection of Serum PCT, hs-CRP and Whole Blood WBC in Acute Upper Respiratory Tract Infection in Children. Modern Medicine and Health Research (Electronic Edition), 4, 146-147.

[23] Sun, C.Y. (2020) Detection and Analysis of Pathogens of Acute Upper Respiratory Tract Infections in Children. Digest of World Latest Medical Information (Conti- 
nuous Electronic Journal), 20, 242-243.

[24] Xiao, J., Ying, J. and Li, M. (2019) Analysis of Pathogenic Bacteria Distribution and Drug Resistance of Acute Respiratory Infections in Children and the Value of Auxiliary Diagnosis of PTC and IL-6 Expression Levels. China Maternal and Child Health Care, 34, 4243-4245.

[25] Chen, X.J., Liu, Z.Q., Wu, X.C., et al. (2019) Distribution Characteristics of Pathogens in Acute Respiratory Infections in Children. Diet and Health Care, 6, 8-9.

[26] Tang, J. (2020) Analysis of the Application of Nine Joint Inspections of Respiratory Pathogens in Children with Acute Respiratory Diseases. The World s Latest Medical Information Abstracts (Continuous Electronic Journals), 20, 64-65.

[27] Miao, C.G. (2019) Analysis of the Rationality of Clinical Medication in 60 Children with Acute Upper Respiratory Tract Infection. Anti-Infective Pharmacy, 16, 11481150 .

[28] Qian, H.C. (2020) Clinical Observation of Yinqiao Powder Combined with Western Medicine in the Treatment of Fever in Children with Acute Upper Respiratory Tract Infection. Electronic Journal of Integrated Traditional Chinese and Western Medicine Cardiovascular Diseases, 8, 182, 186.

[29] Ma, Y.N., Hu, S.Y., Qiu, Y.P., et al. (2020) Meta Analysis of Xiaoer Liaoqiao Qingre Granules versus Ribavirin in the Treatment of Acute Upper Respiratory Tract Infections in Children. Asia-Pacific Traditional Medicine, 16, 177-181.

[30] He, J.J. (2020) The Application Effect of Reduning Injection in the Treatment of Children with Acute Upper Respiratory Tract Infection with Fever. Wisdom Health, 6, 175-177.

[31] Lu, G.R. (2020) Explore the Clinical Effect of Pudilan Xiaoyan Oral Liquid in the Treatment of Acute Upper Respiratory Tract Infection in Children. Wisdom Health, 6, 185-186.

[32] Tang, C.M., Sui, H.H., Zhao, H.X., et al. (2020) Study on the Rationality of Clinical Medication for Acute Upper Respiratory Tract Infection in Children. Health Must Read, No. 9, 68-69.

[33] Wang, Y.Y. and Zhang, L.G. (2020) Clinical Study of Xiaoer Jiegan Granules Combined with Cefuroxime Axetil in the Treatment of Acute Upper Respiratory Tract Infections in Children. Modern Medicine and Clinics, 35, 1659-1662.

[34] Tao, B.Q. and Wang, J.Q. (2021) Efficacy and Safety of Jinlianhua Granules Combined with Antibiotics in the Treatment of Children with Acute Respiratory Infections. Journal of Rare and Uncommon Diseases, 28, 21-22.

[35] Qian, Q.F. (2020) Comparison of the Effects of Azithromycin and Cefuroxime in the Treatment of Acute Bacterial Upper Respiratory Tract Infections in Children. Contemporary Medical Review, 18, 146-147.

[36] Li, H.B. and Liao, Y.Q. (2020) Study on the Effect of Comfort Nursing in the Nursing of Children with Acute Upper Respiratory Tract Infection. Special Health, No. $16,186$.

[37] Cui, J.M. (2020) Xiaoer Kechuanling Oral Liquid (Concentrated Type) in the Treatment of Children with Acute Upper Respiratory Tract Infection Clinical Research. Jiayou Yunbao, 2, 17.

[38] Chen, L. (2020) Study on the Rationality of Clinical Medication for Acute Upper Respiratory Tract Infection in Children. Electronic Journal of Clinical Medicine Literature, 7, 15-16.

[39] Lu, Y.P., Cui, X.Y., Zhang, L., et al. (2020) Clinical Test Results of Mycoplasma 
Bronchopneumonia Infection in Children. Medicine Dietetics and Health, 18, 187188.

[40] Chen, J. and Yuan, C.Y. (2020) Analysis of Clinical Test Results of 40 Children with Mycoplasma pneumoniae Infection. Diet and Health Care, 7, 113-114.

[41] Lin, M.C., Li, X.J. and Li, D.F. (2020) Analysis of Detection Results of Seven Respiratory Viruses in Children. Chinese and Foreign Medical Care, 39, 177-179, 191. 